\title{
Morphological variants of pes anserinus in South India
}

\author{
B.V. Murlimanju, R. Vadgaonkar, C. Ganesh Kumar, M.D. Prameela, M. Tonse, \\ M. M. Pai, V. Blossom
}

Department of Anatomy, Kasturba Medical College, Mangalore-575004, Manipal Academy of Higher Education, Manipal, Karnataka, India.

\author{
CORRESPONDING AUTHOR: \\ Murlimanju Bukkambudhi \\ Virupakshamurthy \\ Department of Anatomy, Kasturba \\ Medical College, \\ Manipal Academy of Higher Education, \\ Mangalore - 575004, India. \\ Phone: +91 8242211746 \\ E-mail: flutemist@gmail.com \\ DOI: \\ 10.32098/mltj.03.2019.11
}

LEVEL OF EVIDENCE: 1B

\begin{abstract}
SUMMARY
Background. It was described that variations exist in the pes anserinus tendons. In this study, our aim was to observe the variations of the pes anserinus in South Indians.

Methods. We examined 53 embalmed human adult lower extremities. The pes anserinus was macroscopically observed for the morphological variants after dissecting the tendons which inserted at the antero medial surface of proximal tibia.

Results. The pes anserinus was formed by 3 tendons, gracilis, semitendinosus and sartorius in 48 lower limbs $(90.6 \%)$. It was observed that, $41(77.4 \%)$ had convergent type, 7 $(13.2 \%)$ had early convergence and 5 lower limbs $(9.4 \%)$ were having divergent pattern of pes anserinus morphology. Semimembranosus muscle was taking in the formation of pes anserinus in 3 lower limbs $(5.7 \%)$. The pes anserinus was inserting into the fascia cruris and not tibia in 8 specimens $(15.1 \%)$. However in 10 cases $(18.9 \%)$, the insertion was both at the tibia and fascia cruris. The insertion was only at fascia cruris and not in tibia in $15.1 \%$ cases. The semitendinosus was giving an extra slip, which was attaching to the medial condyle of tibia in 7 cases (13.2\%). It was giving slip to medial collateral ligament in 2 cases $(3.8 \%)$ and ligamentum patellae in 1 case $(1.9 \%)$.

Conclusion. We believe that this information about morphological variants of pes anserinus is helpful to the plastic and orthopaedic surgeons. Preoperative radiological examination of pes anserinus may be helpful to the operating surgeons to avoid the subsequent difficulties during the procedures like transplant surgeries and graft harvesting during reconstructive surgeries.
\end{abstract}

\section{KEY WORDS}

knee; muscles; tibia; transplants

\section{BACKGROUND}

The pes anserinus is compoundly formed tendon present at medial aspect of knee joint and leg (1-3). The radiating arrangement of tendons resemble 'goose foot', hence the name 'pes anserinus' $(1,2)$. Pes anserinus is sometimes known as 'guy ropes'. This pes anserinus is usually formed by three muscle tendons, namely sartorius, gracilis and semitendinosus2. But it has been reported that there are other musculotendinous structures involved in its formation $(1,2)$. The tendons radiate during the insertion and it has been reported that the variations exist in the tendons. The pes anserinus muscles behave as knee joint flexors and medial rotators of tibia, which act maximally at $90^{\circ}$ flexion. The lateral rotation of tibia is prevents the lateral rotation of the knee joint 4 . Semitendinosus is powerful among the muscles forming the pes anserinus. The pes anserinus transplant is being performed for the unhappy triad of the knee joint. The unhappy triad includes the ruptures of tibial collateral ligament, medial meniscus and anterior cruciate ligament. The transplants of pes anserinus are required for balancing the knee joint and preventing lateral rotation during procedures like repair of dislocated patella (4). It was reported that the pes anserinus transplant can control the rotatory instability and prevent the abnormal valgus deformity as well as the lateral rotation of tibia $(5,6)$.

The dissection of pes anserinus tendons is very important in the knee joint reconstructive procedures (7). The Gracilis and semitendinosus have been commonly used for the 
reconstruction of intracapsular ligaments (8). The morphological knowledge of pes anserinus is also important in the steroid injection procedure for the anserine bursitis (9). It has been reported that, in few patients the flexion and extension of the knee joint revealed an audible pop sound along with the palpable painful snapping sensation (10). The ultrasound examination in these patients demonstrated the change in the position of pes anserinus tendons (10). Due to all these clinical implications, the present study was undertaken. There are only a few papers available about this subject globally, but the same are not available from the Indian subjects. In this context, the present anatomical investigation aimed to study the variant morphology of the pes anserinus in South Indians.

\section{MATERIALS AND METHODS}

In this cadaveric study, 53 formalin fixed human adult lower extremities were utilized. The side and gender of the lower limb specimen were not taken into consideration. The lower limbs which exhibited pathological changes and damages were not considered as part of this study. The lower limbs were kept in supine position. Then a vertical midline incision was given from $5 \mathrm{~cm}$ above the knee joint to $10 \mathrm{~cm}$ below the knee joint. Horizontal incisions were given medially at the superior and inferior limit of the vertical incision. The pes anserinus was exposed by reflecting the skin and fascia. The tendons inserting at proximal tibia, at the anteromedial surface were carefully identified with the meticulous dissection. The tendons forming the guy ropes, semitendinosus, gracilis and sartorius were exposed. The morphological variants of the pes anserinus like additional slips and variant tendons were carefully observed macroscopically and the pictures were taken. The variants were analysed and the frequency was calculated. The present study has the approval of institutional ethics committee of our institution. We state that this present anatomical research was conducted according to international ethical standards, which are required by the journal as per the opinion of Padulo et al. (11).

\section{RESULTS}

\section{Descriptive analysis of pes anserinus}

\section{Constituting tendons}

The pes anserinus was formed by 3 tendons, gracilis, semitendinosus and sartorius in 48 lower limbs $(90.6 \%)$. In this pattern, gracilis tendon was in between the semitendinosus and sartorius tendons (Figure 1). Semimembranosus muscle was taking in the formation of pes anserinus (Figure 2) in only 3 lower limbs (5.7\%). The gracilis and sartorius were absent in one lower limb each $(3.8 \%)$. The frequency of the tendons which took part in the formation of pes anserinus in this study are summarized in Figure 3.

\section{Tendon convergence pattern}

The frequency of tendon convergence pattern is represented in Figure 4. It was observed that, 41 lower limbs $(77.4 \%)$ had convergent type of pes anserinus. In 7 cases (13.2\%), semitendinosus was giving an extra slip (Figure 5), which was attaching to the medial condyle of tibia (early convergence type). The remaining 5 lower limbs $(9.4 \%)$ were having divergent pattern (Figure 4).



Figure 1. Usual type of pes anserinus in which the gracilis tendon $(G)$ is in between the semitendinosus (ST) and sartorius $(S)$ tendons and all joined each other together (90.6\%).

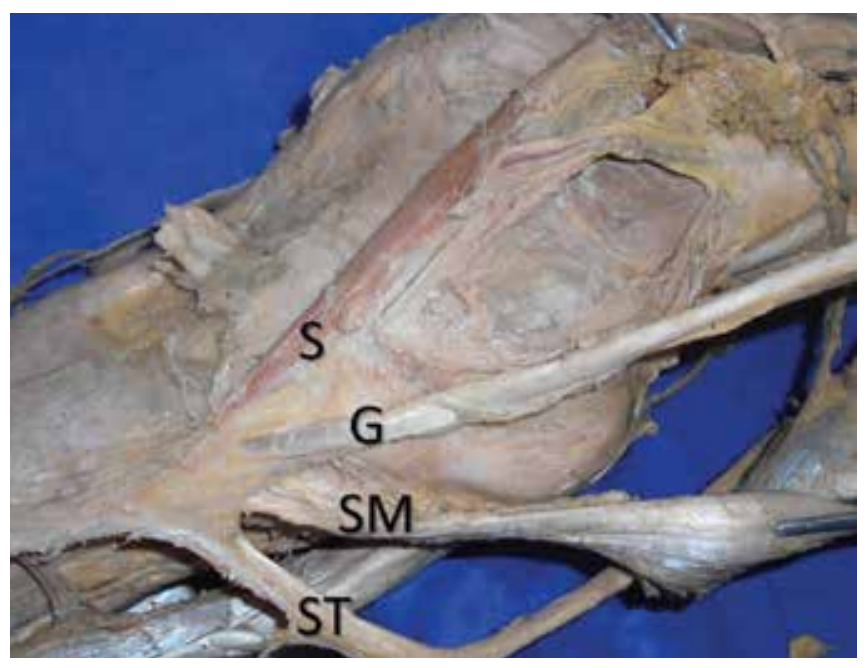

Figure 2. Semimembranosus muscle (SM) taking part in the formation of pes anserinus (5.7\%). 


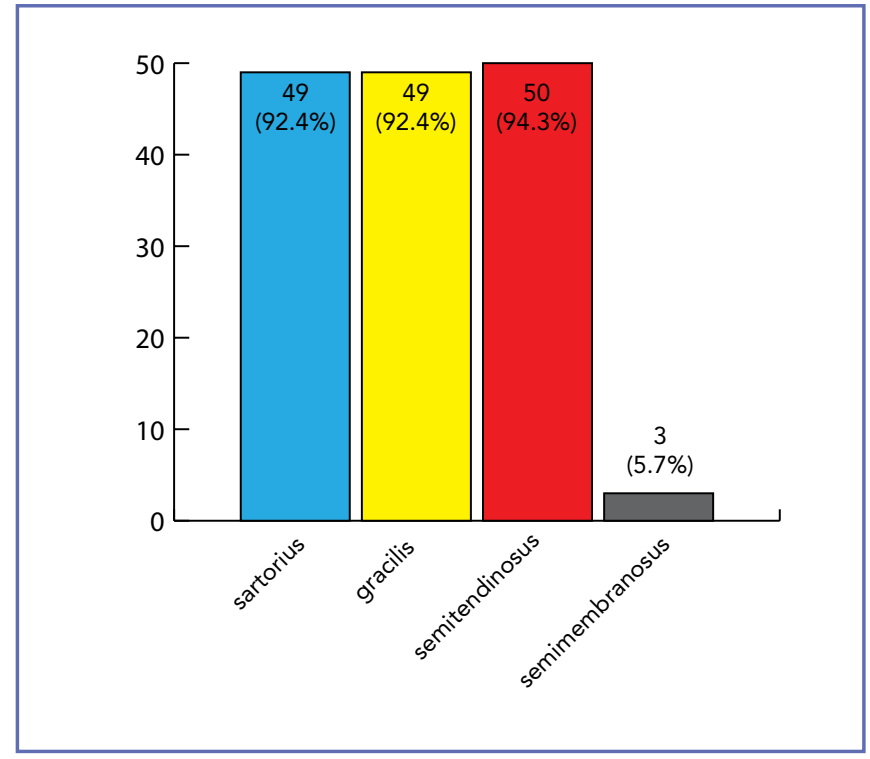

Figure 3. The frequency of tendons which took part in the formation of pes anserinus



Figure 4. Frequency of morphological distribution of convergence of pes anserinus $(n=53)$

\section{Mode of insertion}

The pes anserinus was giving insertion at the superomedial aspect of proximal tibia in 32 lower limbs $(60.4 \%)$. It was inserting into the fascia cruris and not tibia (Figure 6) in 8 specimens $(15.1 \%)$. However in 10 cases $(18.9 \%)$, the



Figure 5. Semitendinosus giving an extra slip (early convergent type), which is attaching to the medial condyle of tibia (13.2\%).

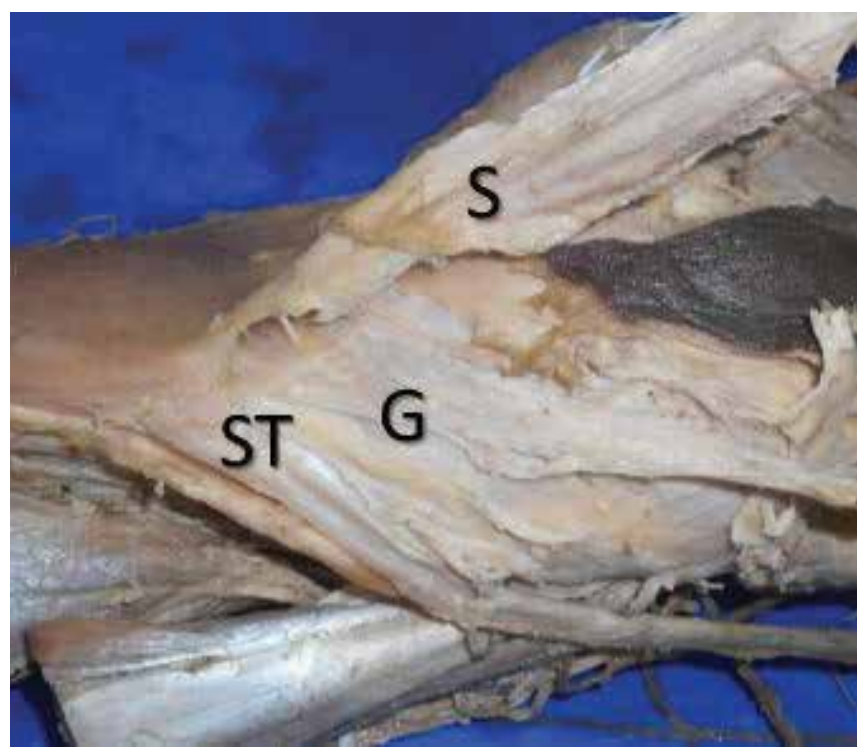

Figure 6. Pes anserinus inserting into the fascia cruris instead of tibia (15.1\%).

insertion was both at the tibia and fascia cruris. The pes anserinus was also giving slip to medial collateral ligament in 2 cases $(3.8 \%)$ and ligamentum patellae in 1 case $(1.9 \%)$. The frequency of various mode of insertion of pes anserinus of this study are summarized in Figure 7. 


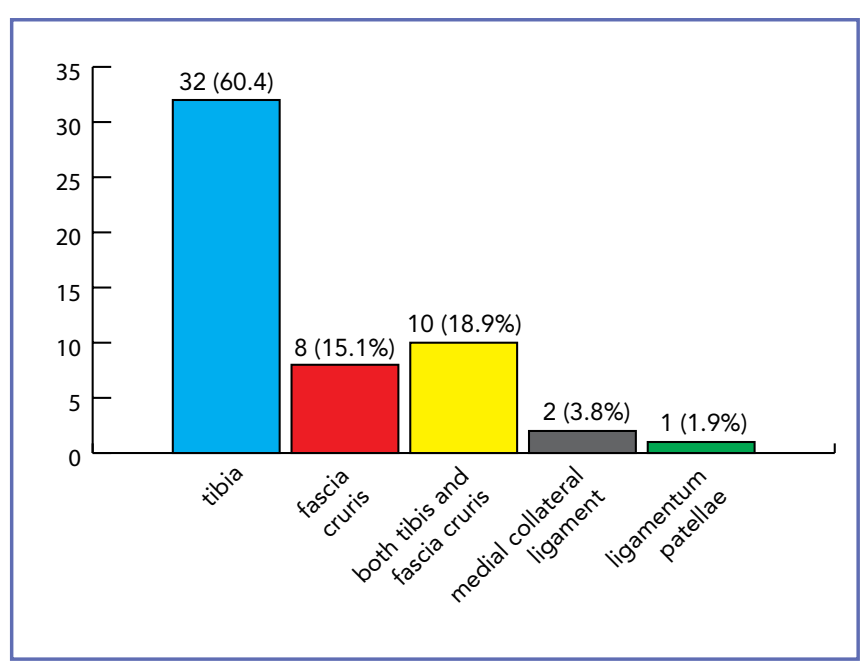

Figure 7. The frequency of various mode of insertion of pes anserinus of this study

\section{Global wise comparison of data of the present study}

The data of the present study have been compared with the previous reports from the other population groups globally. The comparison was performed with respect to the constituting tendons of pes anserinus, convergence pattern and mode of insertion. The descriptive statistics of the comparison are given in tables I-III.

\section{DISCUSSION}

The pes anserinus is conjointly formed by three muscles, each one of it represents each compartment of the thigh. Sartorius muscle is the longest muscle in the body, which takes origin from the anterior superior iliac spine of the hip and belongs to the muscle of front of thigh. Gracilis is known as 'custodian of virginity' which originates from ischiopubic ramus and represents the medial compartment of thigh. Semitendinosus is a hamstring muscle which is attached to the ischial tuberosity and located at the back of thigh (12). Sartorius, gracilis and semitendinosus muscles receive their innervation from the femoral nerve, obturator nerve and tibial nerve respectively. These muscles get the arterial supply from the muscular branches of the femoral, profunda femoral, medial circumflex femoral, inferior gluteal, and perforating arteries (12).

Sartorius performs flexion of both hip and knee joints. It also causes hip abduction and lateral rotation. The action of gracilis would lead to adduction, medial rotation and flexion of thigh. Semitendinosus muscle causes extension of the hip and flexion of knee along with the medial rotation of leg. These muscles are known as 'guy ropes', because they organize into a tent like arrangement, which offers strength and stability to the pelvis. Guy ropes help in weight distribution and balancing of lower extremity movements. The pes anserinus muscles share a common insertion, though the origin, anatomical position, function, nerve supply and blood supply are different for the three muscles. It has been reported that the morphological knowledge of the tendons forming the pes anserinus is clinically important to utilize the correct size of tendon grafts ${ }^{13}$. Cidambi et al. (14) reported that low-lying muscle belly can be seen in the pes anserinus and accessory tendon may be observed while harvesting the gracilis tendon. It was reported that the accessory tendon was seen in $2.4 \%$ of cases (14). The present study observed variant pattern in $9.4 \%$ of cases. This is higher prevalence in comparison to Cidambi et al. (14) (table I). They reported in their radiological study that the anomalous muscle could be sartorius, gracilis or semimembranosus. In this present South Indian study, it was observed that the pes anserinus is formed by three tendons, sartorius, gracilis and semitendinosus in $90.6 \%$ cases (table 1). This prevalence is almost similar to the findings of $97.6 \%$ in North Indian study by Cidambi et al. (14). This morphological type had prevalence of $52.9 \%$ in a Polish study by Olewnik et al. (15). It is interesting to see that Ashaolu et al. (3) observed this pattern in only 5\% cases. But in this Nigerian study, only 20 lower limb specimens were studied. In the present study, semimembranosus muscle was taking part in the formation of pes anserinus in $5.7 \%$ of cases.

Table I. Population wise comparison of morphological variants of tendons of pes anserinus. Pes-pes anserinus; S-sartorius; G-Gracilis; ST-semitendinosus; SM-semimembranosus

\begin{tabular}{lllll}
\hline Authors & Ashaolu et al. (2015) & Cidambi et al. (2016) & Olewnik et al. (2018) & Present study \\
\hline $\mathrm{n}$ & 20 & 123 & 102 & 53 \\
\hline population & Nigerian & North American & Polish & South Indian \\
\hline Pes formed by S/G/ST & $5 \%$ & $97.60 \%$ & $52.90 \%$ & $90.60 \%$ \\
\hline Pes formed by S/G/ST/SM & $25 \%$ & - & - & $5.70 \%$ \\
\hline other variants & $70 \%$ & $2.40 \%$ & $47.10 \%$ & $3.70 \%$ \\
\hline
\end{tabular}


The semimebranosus taking part in the formation of pes anserinus was high $(25 \%)$ in Nigerian population (table I) as per study by Ashaolu et al. (3) The pes anserinus had other morphological variants in its formation with the prevalence rate of $70 \%$ in Nigerians (3), $2.4 \%$ in North Americans (14), $47.1 \%$ in Polish (15) and 3.7\% in the present study. Our findings support the opinion of North American study by Cidambi et al. (14) in this aspect (table I). It was also identified that, semitendinosus was giving an extra slip which was attaching to the medial condyle of tibia in $13.2 \%$ of cases (table III). This was not observed by Cidambi et al. (14) in North Americans, however this is observed in various populations like Koreans (9) (34\%), Polish (15) $(47.1 \%)$ and very high prevalence of $90 \%$ in Nigerians (3) (table III).

Ashaolu et al. (3), in their study from Nigerian population observed that only $85 \%$ of their specimens had usual morphology of the convergent pattern of 3 tendons. In our study, this was observed in $77.4 \%$ cases. This difference is due to the consideration of minute variations like additional slip of the pes anserinus, which were not included as usual by Ashaolu et al. (3). In the present South Indian study, the early convergent pattern was observed in $13.2 \%$ cases and in a Nigerian study by Ashaolu et al. (3), this was observed in $5 \%$ cases (table II). In the present study medial collateral ligament was blended with the pes anserinus in 3.8\% cases (table III). This is almost same with Nigerian study (3) $(5 \%)$. In their study, there was accessory slip from the semitendinosus tendon in $65 \%$ cases and here we observed it in only $13.2 \%$ cases. We observed divergent variety of pes anserinus in $9.4 \%$ cases, which is almost the prevalence rate of $10 \%$ in a study from Nigerian population3 (table II). Ashaolu et al. (3) reported the combined insertion of pes anserinus to both the tibia and fascia cruris in only $10 \%$ cases. Our South Indian study observed this morphological variant in $18.9 \%$ cases (table III). The difference in the incidence of this variants with respect to Indian and Nigerian populations may also be due to racial variations. In a Brazilian study by Munhoz et al. (16), it was reported that there were no morphological variations in the pes anserinus formation. But this study has examined only 7 lower limbs, which was very small sample size. The findings of our present anatomical investigation cannot be compared with this Brazilian study.

Cidambi et al. (14) reported that the operating surgeons should think of a variant tendon morphology, when the tendon is observed to have a low lying muscle belly during harvesting. The surgical excision of pes anserinus is a common practice in the constructive procedures like repair of tendocalcaneus, repair of ruptured patellar tendon and its subluxation, anterior cruciate ligament reconstruction and pes anserinus transplantation during surgical grafting1-3. The painful knee is a common complaint in the orthopaedic practice, it was reported that pes anserinus syndrome has been frequently diagnosed (17). The pes anserinus is the preferred choice for the reconstruc-

Table II. Population wise comparison of convergence pattern of pes anserinus

\begin{tabular}{lll}
\hline Authors & Ashaolu et al. (2015) & Present study \\
\hline $\mathrm{n}$ & 20 & 53 \\
\hline population & Nigerian & South Indian \\
\hline convergent type & $85 \%$ & $77.4 \%$ \\
\hline early convergent & $5 \%$ & $13.2 \%$ \\
\hline divergent & $10 \%$ & $9.4 \%$ \\
\hline
\end{tabular}

Table III. Population wise comparison of insertion pattern of pes anserinus variants

\begin{tabular}{lllll}
\hline Authors & Lee et al. (2014) & Ashaolu et al. (2015) & Olewnik et al. (2018) & Present study \\
\hline $\mathrm{n}$ & 86 & 20 & 102 & 53 \\
\hline population & Korean & Nigerian & Polish & South Indian \\
\hline aST & $34 \%$ & $90 \%$ & $47.1 \%$ & $13.2 \%$ \\
\hline insertion to tibia \& FC & - & $10 \%$ & - & $18.9 \%$ \\
\hline insertion to MCL & - & $5 \%$ & - & $3.8 \%$ \\
\hline
\end{tabular}

aST-accessory slip of semitendinosus; FC-fascia cruris; MCL-medial collateral ligament 
tive surgeries because of the lesser donor site morbidity and no post-operative clinical or functional deficits (18). The anatomical variations should be taken into consideration, while doing the harvesting of tendons. This is the preventive measure which decreases the intraoperative and post-operative complications (19).

The patients with pes anserine bursitis will present with pain and swelling in the medial aspect of the knee joint and may confuse the clinician with the other differential diagnosis of the knee pathologies (20). The accumulation of fluid deep to the tendons and decrease in the echogenicity of tendons during the ultrasound examination are suggestive of tendinopathy (21). Chronic diseases like diabetes mellitus, osteoarthritis, obesity and congenital deformities like genu valgus are the predisposing factors for the pes anserine bursitis and pes anserine tendon disease.

There are only a very few scientific papers about pes anserinus are available in the literature. The novelty of the present study is that, this is the first of the studies conducted in South Indian population. This can be considered as the strength of this anatomical investigation. Earlier reports from other populations just observed if the pes anserinus is convergent or divergent pattern. The present study has observed additional variations like semimembranosus involving in the formation of pes anserinus and the pes anserinus inserting into the medial collateral ligament and medial condyle of tibia. It is believed that these anatomical variations have implications in understanding the pathophysiology of snapping knee syndrome and unhappy triad of the knee joint. The new anatomical variations of the pes anserinus which are observed in this study, which are not reported earlier can be considered as the strengths of this study. These anatomical variations are not only of morphological importance, but also have surgical implications.

\section{CONCLUSION}

In this South Indian anatomical investigation, we report several variants of the pes anserinus, the details of which are important to the operating plastic surgeon and orthopaedic surgeon. The preoperative observation of pes anserinus by using radiological investigations like ultrasound or nuclear magnetic resonance may be the orthopedician and plastic surgeon. This may provide the preoperative evidence of an anomalous tendon of the pes anserinus formation. The data can be used as a morphological database of clinical anatomy of pes anserinus from South Indian population. However, this study has limitation of gender based observation and side based variation, which could not be done. The sample size is small in this study, the findings can be further evaluated with still good number of specimens.

\section{Conflict of interest}

We state that there are no relationships, circumstances and conditions which have potential conflicts of interest associated with this manuscript.

\section{REFERENCES}

1. Amatuzzi MM, Cocco LF, Di Dio LJ, Gotfryd AO. Surgical anatomy of the variations of the arrangement of the tendons of the muscles of the pes anserinus in male adults. Italian J Anat Embryol. 2002;107:29-35.

2. Mochizuki T, Akita K, Muneta T, Sato T. Pes anserinus: layered supportive structure on the medial side of the knee. Clin Anat. 2004:17:50-54.

3. Ashaolu JO, Osinuga TS, Ukwenya VO, Makinde EO, Adekanmbi AJ. Pes anserinus structural framework and constituting tendons are grossly aberrant in Nigerian population. Anat Res Int. 2015:483186.

4. Slocum DB, Larson RL, James SL. Pes anserinus transplant: impressions after a decade of experience. J Sports Med. 1974;2:123-136.

5. Slocum DB, Larson RL. Rotatory instability of the knee. Its pathogenesis and a clinical test to demonstrate its presence. J Bone Joint Surg. 1968;50A:211, 225.

6. Slocum DB, Larson RL. Pes anserinus transplantation. A surgical procedure for control of rotatory instability of the knee. J Bone Joint Surg. 1968;50A:226-242.

7. Ivey M, Prud'homme J. Anatomic variations of the pes anserinus: a cadaver study. Orthopedics. 1993;16:601-606.

8. Kijkunasathian C, Limitlaohaphan C, Saengpetch N, Saitongdee $\mathrm{P}$, Woratanarat $\mathrm{P}$. The location of pes anserinus insertion in Thai people. J Med Assoc Thai. 2009;92 Suppl 6: S189-192.

9. Lee JH, Kim KJ, Jeong YG, et al. Pes anserinus and anserine bursa: anatomical study. Anat Cell Biol. 2014;47:127-131.

10. Rainey CE, Taysom DA, Rosenthal MD. Snapping pes anserine syndrome. J Orthop Sports Phys Ther. 2014;44:41.

11. Padulo J, Oliva F, Frizziero A, Maffulli N. Muscles, Ligaments and Tendons Journal - Basic principles and recommendations in clinical and field Science Research: 2016 Update. Muscles Ligaments Tendons J. 2016;6:1-5.

12. Standring S, ed. Gray's Anatomy: The Anatomical Basis of Clinical Practice, 41st ed. New York; Elsevier Limited. 2015;1361-1366.

13. Jayaram PR, Freeman AJ, Jacobson NA, Fogg QA. Variations of the pes anserinus with potential functional and clinical implications. Int J Surg. 2013;11:601.

14. Cidambi KR, Pennock AT, Dwek JR, Edmonds EW. Avoiding anomalous tendon harvest at the pes anserinus insertion. J Knee Surg. 2016;29:80-83.

15. Olewnik Ł, Gonera B, Podgórski M, Polguj M, Jezierski H, Topol M. A proposal for a new classification of pes anserinus morphology. Knee Surg Sports Traumatol Arthrosc. 2018 Dec 10. doi: 10.1007/s00167-018-5318-3.

16. Munhoz MAS, Cunha FB, Mestriner G, et al. Anatomical and morphometric study of the pes anserine tendons in the knee. Journal of Clinical and Diagnostic Research. 2018;12:RC05RC07. 
17. Milton Helfenstein Jr M, Kuromoto eJ. Anserine syndrome. Bras J Rheumatol. 2010; 50:313-327.

18. Candal-Couto JJ, Deehan DJ. The accessory bands of Gracilis and semitendinosus: an anatomical study. Knee. 2003;10:325-328.

19. Charalambous CP, Kwaees TA. Anatomical considerations in hamstring tendon harvesting for anterior cruciate ligament reconstruction. Muscles Ligaments Tendons J. 2013;2:253-257.
20. Rennie WJ, Saifuddin A. Pes anserine bursitis: incidence in symptomatic knees and clinical presentation. Skeletal Radiol. 2005;34:395-398.

21. Alves TI, Girish G, Kalume Brigido M, Jacobson JA. US of the knee: scanning techniques, pitfalls, and pathologic conditions. Radiographics. 2016; 36: 1759-75. 